\title{
Personal Construct Therapy vs Cognitive Behavioral Therapy in the Treatment of Depression in Women with Fibromyalgia: Study Protocol for a Multicenter Randomized Controlled Trial
}

This article was published in the following Dove Press journal:

Neuropsychiatric Disease and Treatment

\author{
Clara Paz (D) \\ Mari Aguilera $\mathbb{D}^{2}$ \\ Marta Salla (iD ${ }^{3}$ \\ Victoria Compañ (iD) ${ }^{3}$ \\ Joan C Medina (iD ${ }^{3,4}$ \\ Arturo Bados (iD) ${ }^{3}$ \\ Eugeni García-Grau (D) 3 \\ Antoni Castel iD ${ }^{5}$ \\ José Cañete Crespillo (D) ${ }^{6}$ \\ Adrián Montesano (iD) ${ }^{7}$ \\ Leticia Medeiros- \\ Ferreira (iD) ${ }^{8}$ \\ Guillem Feixas (iD) 3,4 \\ 'School of Psychology, Universidad de \\ Las Américas, Quito, Ecuador; \\ ${ }^{2}$ Department of Cognition, Development \\ and Educational Psychology, Faculty of \\ Psychology, Universitat de Barcelona, \\ Barcelona, Spain; ${ }^{3}$ Department of Clinical \\ Psychology and Psychobiology, Faculty of \\ Psychology, Universitat de Barcelona, \\ Barcelona, Spain; ${ }^{4}$ The Institute of \\ Neurosciences, Universitat de Barcelona, \\ Barcelona, Spain; ${ }^{5}$ Hospital Universitari \\ de Tarragona Joan XXIII, Tarragona, \\ Spain; ${ }^{6}$ Department of Psychiatry, \\ Hospital de Mataró, Mataró, Spain; \\ ${ }^{7}$ Faculty of Psychology and Educational \\ Sciences, Universitat Oberta de \\ Catalunya, Barcelona, Spain; ${ }^{8}$ Nou Barris \\ Nord Adult Mental Health Centre, \\ Barcelona, Spain
}

Correspondence: Clara Paz Jose Queri y Av, De Los Granados, Quito ECI70I25, Ecuador

Tel +59323981000 ext. 2518

Email clara.paz@udla.edu.ec
Background: Fibromyalgia (FM) is a debilitating syndrome, more prevalent in women, which is aggravated by the presence of depressive symptoms. In the last decade, cognitive behavioral therapy (CBT) has demonstrated to reduce such depressive symptoms and pain in these patients, but there are still a considerable number of them who do not respond to interventions. The complexity of the disorder requires the consideration of the unique psychological characteristics of each patient to attain good outcomes. One approach that could accomplish this goal might be personal construct therapy (PCT), an idiographic approach that considers identity features and interpersonal meanings as their main target of intervention. Then, the aim of the study is to test the efficacy of PCT as compared to a wellestablished treatment in the reduction of depressive symptoms in women with fibromyalgia. Methods and Analysis: This is a multicenter randomized controlled trial. In each condition participants will attend up to eighteen 1-hr weekly therapy sessions and up to three 1-hr booster sessions during the following 3-5 months after the end of treatment. The depression subscale of the Hospital Anxiety and Depression Scale (HADS-D) will be the primary outcome measure and it will be assessed at baseline, at the end of therapy, and at 6-month follow-up. Other secondary measures will be applied following the same schedule. Participants will be 18- to 70-years-old women with a diagnosis of FM, presenting depressive symptoms evinced by scores above seven in depression items of the HADS-D. Intention-to-treat and complete case analyses will be performed for the main statistical tests. Linear mixed models will be used to analyze and to compare the treatment effects of both conditions.

Trial Registration: ClinicalTrials.gov: NCT02711020.

Keywords: psychotherapy, pain, repertory grid technique, study protocol, CBT, personal construct therapy

\section{Introduction}

Fibromyalgia (FM) is a syndrome characterized by the presence of generalized and chronic musculoskeletal pain. It is the most common pain condition and it is the second most frequent rheumatic disorder. ${ }^{1}$ The worldwide prevalence is approximately $2.7 \%{ }^{2}$ In Spain, the reported prevalence ranged from $2.3 \%$ to $2.7 \%$, and it is more prevalent in females $(3.3-4.2 \%)$ than in males $(0.2-1.3 \%)^{3,4}$

Patients with FM indicate that they experience significantly more intense pain than patients with other pain conditions. ${ }^{5,6} \mathrm{FM}$ has a high comorbidity of psychiatric issues, 
especially of affective disorders. ${ }^{7}$ Major depression disorder seems to be the most frequent diagnosis with a lifetime prevalence ranging from $62 \%$ to $86 \%$, but depressive symptoms are present for $90 \%$ of the patients. ${ }^{8}$ These patients with both FM and depressive symptoms exhibit lower levels of physical functioning and worse quality of life than those without depressive symptoms. ${ }^{9,10}$ The presence of depressive symptoms at the onset of development of the syndrome predicts limited treatment response in terms of pain intensity reduction. ${ }^{11}$ In general, these data confirm that symptoms of depression exacerbate and aggravate the chronic course of the disease; and as a result, patients presenting both conditions consume more health-care resources, even considering the additional cost of each condition when diagnosed separately. ${ }^{12}$

Generally, the accepted objectives for the treatment of FM are to relieve pain, to acquire emotional balance, to improve physical activity, and other associated problems. ${ }^{13,14}$ Nowadays, strong evidence exists in favor of multidisciplinary treatment, which might include medication, education about the syndrome, moderate physical activity and psychological interventions. ${ }^{1,15}$ In relation to psychological interventions, an extensive review conducted by Glombiewski et al ${ }^{16}$ indicates the presence of small effect sizes related to pain reduction, both at short- and long-term measurement. All the included treatments show similar efficacy in the reduction of depressive symptoms, although cognitive behavioral therapy (CBT) presented the greatest effect sizes regarding short-term pain reduction. Another meta-analysis ${ }^{17}$ assessing only CBT supports its effects on improving self-efficacy, pain, and depressive symptoms, but yields small effect sizes for pain reduction and for improving the quality of life.

After several decades of studies about treatments for FM, one of the main limitations which have been recognized is the underestimation of the complexity of the disorder, resulting in a lack of specificity of the treatments. ${ }^{18}$ It is essential to identify which psychological profiles respond to specific treatments. ${ }^{19,20}$ Treatments should adapt to the needs and specific psychological features of the patients, taking into consideration their physical functioning and, especially, their depressive symptoms. ${ }^{13,21,22}$ Although brief structured group therapy has been the predominant psychological treatment for FM, individual psychotherapy seems to produce greater effects for this population. ${ }^{21}$ Furthermore, long-term psychological treatments display bigger effect sizes for pain reduction and improvement of depressive symptoms. ${ }^{16}$ Overall, long-term psychological treatments that take into account the unique psychological characteristics $\left[\mathrm{eg}^{23,24}\right]$ of each patient might be in a better position to attain good outcomes. Also, the focus on specific behaviors, thoughts and emotions that characterizes CBT has proved insufficient in its efficacy and we expect that a therapeutic approach addressing the impact on the person's self-identity of developing an FM syndrome might be more efficacious at least in decreasing depressive symptoms. One approach that could accomplish this goal might be personal construct therapy (PCT), ${ }^{23,24}$ an idiographic approach that considers identity features and interpersonal meanings as their main target of intervention. Every person construes (interprets) events in different manners according to their own cognitive system. ${ }^{25}$ PCT is aimed at reconstructing meanings about self, significant others, and relevant events. Several meta-analyses show some favorable evidence with respect to its efficacy. ${ }^{26,27}$ It produces better outcomes than waiting list and usual care conditions, but results are equivalent when compared with active treatments. Also, at follow-up, patients who were treated with PCT showed better results than those who did not receive any active treatment.

PCT has devised assessment tools to assess people's construction of self and others. One of these instruments is the repertory grid technique (RGT). ${ }^{28,29}$ This instrument allows assessing self-concept and cognitive structure based on the interviewee's evaluation of their self, their significant others, and their ideal-self by means of their own personal constructs. Personal constructs are bipolar discriminations (eg, "happy vs sad") of how individuals describe themselves and others. ${ }^{23}$ The person's ratings of the self, ideal-self and others (here termed elements in columns) according to their personal constructs (in rows) form a data matrix, each cell reflecting the rating of each element with regard to each construct using a seven-point Likert scale. Although RGT emphasizes idiographic information (i.e., the constructs elicited from the person and their application to the elements), some general cognitive measures can be obtained, including current-self and ideal-self discrepancy, polarized and/or unidimensional construing, and the presence (and number) of cognitive conflicts. RGT has already been applied to understand the personal constructions of patients with depression ${ }^{30}$ and of those with FM, ${ }^{31,32}$ among other conditions ${ }^{33}$. Patients with depression showed negative self-evaluation, perceived isolation and evaluate others negatively. In addition, they present a greater proportion of implicative dilemmas, a type of cognitive conflict in which the symptom is associated with positive personal characteristics of the identity. ${ }^{34}$ Similarly, for women with FM, we found higher self-ideal discrepancy, as well as a more negative evaluation of others, as compared to controls. Moreover, $76.7 \%$ of the clinical sample presented implicative dilemmas, compared with $46.6 \%$ of the control 
group. ${ }^{31}$ When assessing the cognitive structure of patients presenting both, depression and FM, the results indicated that these patients presented greater cognitive rigidity and lower differentiation than patients with depression without FM. ${ }^{32}$

The application of PCT for fibromyalgia using RGT information to conduct the therapy has been tested in a pilot study with 20 women with FM. ${ }^{35}$ Higher improvement was observed in those with a history of maltreatment. Interestingly, these women also had a greater depressive symptomatology at the beginning of therapy. One ingredient of PCT is the work aimed at the resolution of implicative dilemmas, ${ }^{36,37}$ the efficacy of this intervention for depression has been tested in a randomized controlled trial. ${ }^{38}$ The results showed that the inclusion of an individual dilemma focused intervention to group CBT intervention produced a big effect size for the reduction of depressive symptoms (Cohen's $d=1.13$ ), and this effect was comparable with the intervention with group CBT plus individual CBT (Cohen's $d=1.02) .{ }^{39}$

The research question we want to address in this study refers to the efficacy of PCT as compared to a well-established treatment in the reduction of depressive symptoms in women with fibromyalgia. CBT has been chosen as the comparison as it is the most studied psychological treatment ${ }^{16}$ and it is recommended as a treatment of choice in all the reviews and clinical guidelines. ${ }^{1,15}$ Given the promising evidence of a previous pilot study, ${ }^{35}$ we expect that PCT will be more efficacious in the reduction of depressive symptoms in women with FM.

\section{Specific Objectives}

- To determine whether or not PCT is more efficacious than CBT in the reduction of depressive symptoms of women with FM.

- To compare the impact of PCT and CBT on variables such as functional capacity, well-being and quality of life of women with FM.

- To contrast the change in cognitive features (eg, cognitive conflicts, polarization) assessed with the RGT between both therapy conditions.

- To identify the baseline variables which predict common and differential outcomes between both treatments.

\section{Methods and Analysis Design}

This is a multicenter randomized, controlled parallel group, two-arm, superiority trial to compare the efficacy of individual
PCT with individual CBT for reduction of depressive symptoms in women with FM. The allocation ratio for this trial is $1: 1$ (see Figure 1 for trial design). This design not only permits the comparison between treatments but also allows identification of the personal characteristics of those patients who attain better outcomes.

\section{Settings}

This study is conducted at nine mental health centers, with different levels of attention: Nou Barris Nord Mental Health Center (CSMA Nou Barris Nord), Hospital de Mataró, and primary care centers such as CAP Manso (Institut Català de la Salut, Barcelona), CAP Les Hortes (EAP PobleSec), and five primary care centers in Badalona (Badalona Serveis Assistencials).

\section{Participants}

Only female adult patients diagnosed with fibromyalgia by professionals of the health system are included because data show a small percentage of males diagnosed with $\mathrm{FM}^{3,4}$ and, therefore, it is easier to contact and identify female patients within the centers. The decision was taken to maintain the homogeneity of the sample for the statistical analyses. ${ }^{4}$

\section{Inclusion Criteria}

Participants will be women with a diagnosis of FM and depressive symptoms as evinced by a score above seven in the depression items of the Hospital Anxiety and Depression Scale (HADS-D). ${ }^{40}$ The age of participants will range from 18 to 70 years, since this range concentrates most of the incidence of FM patients. ${ }^{4}$

\section{Exclusion Criteria}

Patients are excluded from the study if they present bipolar disorders, psychotic symptoms, substance abuse, acute suicidal ideation, intellectual disability or organic brain dysfunction. Also, patients are not accepted if they are receiving psychological treatment, unless it is suspended (agreement with the patient and their therapist) at the time of inclusion in the study. Finally, those who do not have enough level of competence to communicate in Spanish or Catalan, or patients with substantial visual, hearing and cognitive deficits are not included. The comorbid occurrence of other conditions and consumption of psychotropic drugs are assessed and recorded for statistical control, but they are not causes for exclusion.

The sample size for this study was calculated with regard to the mean comparison between treatment conditions considering an effect size of at least 0.30 to accept the alternative hypothesis 




Figure I Flow chart diagram of the trial design.

Abbreviations: PCT, Personal Construct Therapy; CBT, Cognitive Behavioral Therapy; HADS-D, Hospital Anxiety and Depression Scale - Depression; HADS-A, Hospital Anxiety and Depression Scale - Anxiety; HDRS, Hamilton Depression Rating Scale; FIQ, Fibromyalgia Impact Questionnaire; RGT, Repertory Grid Technique; CORE-OM, Clinical Outcomes in Routine Evaluation - Outcome Measure.

that PCT significantly reduces depressive symptoms in comparison to CBT. With a statistical power of 0.80 , a Type 1 error level of 5\% (one-sided), assuming an estimated common standard deviation of 1 , and a correlation between measurements of 0.85; a total sample of 90 patients is required, 45 patients in each condition. We expect to have a dropout rate of $20 \%$, then a total of 112 patients will be recruited (56 in each condition).

\section{Participant Allocation}

Once the baseline assessment is completed, women who meet the criteria to be part of the study are randomly allocated to one of the two treatment conditions (CBT or PCT). The randomization is performed by a staff member of the
Universitat de Barcelona (not involved in the trial and blind to treatment conditions) using a computer-generated allocation permuted block sequence, stratified by type of center (mental health/primary care) in which the participants are recruited. Treatments are conducted in same center in which the participant receives treatment for FM.

\section{Treatments}

Two individual-format psychological treatments are applied, with a limit of eighteen 1-hr sessions. A maximum of three 1-hr booster sessions can be done according to each patient's needs in the 3-5 months following the end of the treatment. 
Both interventions are based on case conceptualization; then, the number of sessions rely on the goals set up for each patient by their assigned therapist. Contents of session, techniques, and procedures depend on the applied condition, as follows.

\section{Cognitive Behavioral Therapy (CBT)}

The applied techniques and interventions for this condition are adaptations of methods already used with FM patients in the CBT group format:

\section{Psychoeducation}

It includes information about FM, different means to coping with the condition and the influence of depression and anxiety, muscular tension, inactivity, and distorted thinking in the genesis and maintenance of pain. ${ }^{41}$

\section{Relaxation}

Techniques such as diaphragmatic breathing and progressive muscle relaxation are offered to cope with pain.

\section{Behavioral Activation}

Different elements about behavior are managed considering the relation between inactivity, emotions and thoughts associated to depression and FM. To produce activation of the patients, behavioral records are used in companion to task prioritization and gradual involvement in pleasant activities. $^{42,43}$

\section{Cognitive Restructuring}

This module focuses on the relationship between thoughts, emotions and behaviors. The emphasis is on the link between emotions and body reactions. Also in the identification and modification of automatic thoughts and beliefs that are related with depression and FM. ${ }^{43}$

The order and the degree in which these modules are applied varies in order to adapt to the needs of every patient.

\section{Personal Construct Therapy}

The protocol for this condition was specifically designed for the present trial based on previous literature regarding case formulation according to $\mathrm{TCP}^{44}$ and our prior experience in a pilot study. ${ }^{35}$ Four possible courses of action with specific objectives and techniques have been defined from common motives for consultation presented in the pilot study and observed in our clinical practice. In the first two sessions, therapists explore the patients' demands or complaints, their theory of the problem, and the possible solutions they have thought of. Our treatment model considers that patients evolve along the therapy; therefore, it is reasonable to address more than one course of action.

\section{Complaints Focused on Pain}

In these cases, patients' narratives revolve around the symptoms of the FM and, generally, they do not recognize any other problem. The objective of the intervention is to enrich and to nuance the construct "pain-without pain", as well as to promote the inclusion of other constructs in the explanation of the events and in the construction of their personal and corporal experience. The techniques used with them are mainly the psychoeducation with regard to gate control theory of pain ${ }^{45}$ and the externalization of pain using letters to the pain or draws.

\section{Complaints Focused on Emotional Avoidance}

In these cases, the expressed wish is "to stop feeling". Taking into consideration the construing processes involved in this complaint, we conceptualize it as a process of recursive self-invalidation of the system, ${ }^{24}$ in which the activation of a specific emotion (specially anger and sadness) supposes the invalidation of identity aspects of the self. The objective is to revise the meaning-construction system in such a way that emotions are acceptable, compatible rather than invalidating for the self-identity system. The techniques used include the explanation of the dilemma (psychoeducation about the emotions); the historical revision of the emotional dilemma, hypnosis, etc.

\section{Complaints Focused on Interpersonal Conflicts}

These conflicts can be related to FM or to other issues. By exploring the patients' views about these problems, often some difficulties in sociality, ${ }^{23}$ which refers to their capacity to construe the construction processes of another person in a meaningful way, can be observed. The objective is to extend the meaning-construction system in such a manner that it can offer possible action courses that are compatible with the sense of identity of the patient. Possible techniques to increase sociality include representations of the family as sculptures; ${ }^{46}$ use of analogical resources such as metaphors; and the inclusion of some members of the family in some of the sessions, etc.

\section{Ambivalent Complaints About Change}

In these cases, the therapist has identified the presence of one or more implicative dilemmas in the RGT of the participant. $^{47}$ The change has ambivalent implications, which means that a change movement towards the desired pole of the construct (eg, "being happy" instead of "being 
sad") implies a significant change in other constructs that are congruent with the personal identity (eg, "being happy" implies to "be selfish" instead of "generous"). The objective is to resolve the dilemma, so the desired change does not imply a threat to the sense of identity. The intervention will follow the guidelines provided by Feixas and Compañ. ${ }^{37}$

\section{Treatment Integrity}

Both treatment conditions will be conducted by psychotherapists trained in the specific treatment condition that they apply. However, before to be part of the trial, all therapists will attend to a specific training in the protocols used in this study. Senior therapists with at least 10 years of clinical experience will carry out the training; also, they will supervise the psychotherapeutic process and the adherence to intervention protocols for each treatment condition. All therapy sessions will be recorded to verify treatment integrity and to supervise the development of the psychotherapeutic process. The therapist or supervisor will report to the research team any deviation of the intervention protocol for recording in the database. After the completion of the study, the content of $10 \%$ of the total number sessions for each condition will be randomly selected to be monitored and assessed in order to verify the adherence to the guidelines for each specific treatment condition. The conclusions of this assessment will be included in the report of the results of the trial.

\section{Measures}

\section{Primary Outcome Measure}

Depression Items of the Hospital Anxiety and Depression Scale (HADS-D) ${ }^{40,48}$

To assess the symptoms of depression other than somatic problems such as insomnia or fatigue, which can be present in FM patients. It has seven items related to depression, and they are rated in a four-point Likert scale. This instrument has acceptable psychometric properties ${ }^{48}$ and it has shown to be useful with FM patients. ${ }^{49-51}$ The anxiety items of the HADS will be considered as a secondary outcome measure.

\section{Secondary Outcome measures}

Hamilton Depression Rating Scale (HDRS) ${ }^{52,53}$

To evaluate the severity of depressive symptoms, it is rated by the clinician after a clinical interview. The scale contains 17 items, and it has shown good psychometric properties. ${ }^{53}$

\section{Fibromyalgia Impact Questionnaire (FIQ) $)^{54,55}$}

To assess the impact of FM on physical functioning, development of daily tasks, and pain-related symptoms. It has shown good psychometric properties and sensitivity to change after treatment. ${ }^{56}$

\section{Anxiety Items of the Hospital Anxiety and Depression Scales (HADS-A) $)^{40,48}$}

To assess the symptoms of anxiety in FM patients avoiding dependency on somatic aspects.

\section{Visual Analogue Scale for Pain (VAS Pain) ${ }^{57}$}

To assess the intensity of pain session by session, it is a $100 \mathrm{~mm}$ long horizontal straight line, marked at one end with the label "no pain" and the opposite end with the label "worst possible and unbearable pain". Scores range from 0 to 100 . This scale is widely used in clinical practice and research. ${ }^{58}$

\section{Clinical Outcomes in Routine Evaluation - Outcome Measure (CORE-OM) ${ }^{59,60}$}

This is a 34-item self-reported questionnaire designed to assess the change in therapy (pre-post) which evaluates four dimensions: subjective well-being, symptoms or problems, life functioning and risk. It has shown good psychometric properties. The brief version of 18 items (CORE-SFB) is used at the beginning of each session.

\section{Repertory Grid Technique (RGT) ${ }^{23,28,29,61}$}

To assess cognitive structure, self-concept and cognitive conflicts, PCT provides this semi-structured interview focused on the elicitation of bipolar personal constructs and their assessment as they are applied to significant others (elements). Then, every element is rated according to each construct using a 7-point Likert scale. The statistical analysis of the resulting data matrix generates cognitive indexes, distances between different perceptions of the self, and number and intensity of cognitive conflicts (if any). The information given by the RGT is useful for planning the psychotherapeutic process conducted by means of the PCT.

\section{Procedure}

Professionals of collaborating centers will be informed about the study and asked to offer participation to the relevant patients. After referral, potential participants are contacted to arrange a date for the initial assessment $\left(t_{1}\right)$. It begins explaining the study to participants and offering them to sign the informed consent. 
After random allocation to treatment, and once the treatment is completed (maximum 18 sessions), the second assessment $\left(t_{2}\right)$ with all the baseline measures will be conducted by the same evaluators who carried out the baseline assessment (whenever possible). A follow-up assessment $\left(t_{3}\right)$ will be conducted 6 months after the termination of the treatment (see Table 1 for a detailed schedule of assessments and interventions according to SPIRIT recommendations ${ }^{62}$ ). The evaluators are blind to the therapeutic condition of the assessed participant. Participant's allocated intervention will not be revealed to evaluators in any circumstance. Treatments will be discontinued in case of participant request.

\section{Data Management}

Participant data files with personal data are stored in a locked filing cabinet at the University of Barcelona accessible only to one member of the research team who supervises data entry and controls the access to the personal data file. Data integrity checks will be conducted to check completeness using spot checks, and consistency will be tested using

Table I SPIRIT Diagram of Assessments at Baseline $\left(t_{1}\right)$, Allocation (0), Weekly Sessions $\left(s_{1}-s_{18}\right)$, Treatment Termination $\left(t_{2}\right)$, Booster Sessions $\left(t_{2+3 m}-t_{2+5 m}\right)$ and 6-Month Follow-Up $\left(t_{3}\right)$

\begin{tabular}{|c|c|c|c|c|c|c|c|c|c|}
\hline \multirow[b]{3}{*}{ TIMEPOINT } & \multicolumn{9}{|c|}{ STUDY PERIOD } \\
\hline & \multirow{2}{*}{$\frac{\text { Baseline }}{t_{1}}$} & \multirow{2}{*}{$\begin{array}{c}\text { Allocation } \\
0\end{array}$} & \multicolumn{6}{|c|}{ Post-allocation } & \multirow{2}{*}{$\frac{\text { Follow-up }}{t_{3}}$} \\
\hline & & & $s_{1}$ & $S_{n \ldots 18}$ & $t_{2}$ & $t_{2+3 m}$ & $t_{2+4 m}$ & $t_{2+5 m}$ & \\
\hline \multicolumn{10}{|l|}{ ENROLMENT: } \\
\hline Eligibility screen & $\mathrm{X}$ & & & & & & & & \\
\hline Informed consent & $X$ & & & & & & & & \\
\hline $\begin{array}{r}\text { Allocation/ } \\
\text { Randomization } \\
\end{array}$ & & $\mathrm{X}$ & & & & & & & \\
\hline \multicolumn{10}{|l|}{ INTERVENTIONS: } \\
\hline$P C T$ & & & & & & $x$ & $x$ & $x$ & \\
\hline CBT & & & & $\rightarrow$ & & $x$ & $x$ & $x$ & \\
\hline \multicolumn{10}{|l|}{ ASSESSMENTS: } \\
\hline $\begin{array}{r}\text { Sociodemographic and } \\
\text { clinical information }\end{array}$ & $x$ & & & & & & & & \\
\hline$H A D S-D$ & $x$ & & & & $x$ & & & & $x$ \\
\hline HADS-A & $x$ & & & & $x$ & & & & $x$ \\
\hline HDRS & $x$ & & & & $x$ & & & & $x$ \\
\hline$F I Q$ & $\mathrm{X}$ & & & & $x$ & & & & $x$ \\
\hline$R G T$ & $\mathrm{X}$ & & & & $X$ & & & & $\mathrm{X}$ \\
\hline CORE-OM & $x$ & & & & $x$ & & & & $x$ \\
\hline CORE-SFB ${ }^{a}$ & & & & $\rightarrow$ & & $x$ & $x$ & $x$ & \\
\hline VAS Pain ${ }^{a}$ & & & & & & $x$ & $x$ & $x$ & \\
\hline
\end{tabular}

Note: ${ }^{\text {a}}$ Questionnaires applied every session.

Abbreviations: PCT, Personal Construct Therapy; CBT, Cognitive Behavioral Therapy; HADS-D, Hospital Anxiety and Depression Scale - Depression; HADS-A, Hospital Anxiety and Depression Scale - Anxiety; HDRS, Hamilton Depression Rating Scale; FIQ, Fibromyalgia Impact Questionnaire; RGT, Repertory Grid Technique; CORE-OM, Clinical Outcomes in Routine Evaluation - Outcome Measure; CORE-SFB, Clinical Outcomes in Routine Evaluation - Short Form B; VAS Pain, Visual Analogue Scale for Pain. 
plausibility checks. The interventions suppose minimal risk for the participants, then there will not be a data monitoring committee for this study. Once completed the study and the integrity checks, data will be made available in a public repository for open access. Participants' privacy will be protected by excluding any identifying personal data according to ethical standards about data sharing.

\section{Statistical Analyses}

Intention-to-treat and complete case analyses will be performed for the main statistical comparisons. Linear mixed models will be used to analyze and to compare the treatment effects of conditions. Baseline measurements, sociodemographic, clinical variables and number of sessions will be entered in the model as covariates, while outcome measurements will be the dependent variables. Potential moderator role of the cognitive measures obtained by the RGT will be explored, especially those reflecting cognitive conflicts. For all the analyses, effect sizes will be calculated. To accept the hypothesis that the PCT is superior than CBT, the effect size when comparing both treatments at the end of the intervention and at follow-up must be at least 0.30 , demonstrating that the reduction of depressive symptoms is greater in PCT than in CBT condition.

CORE-OM will permit to assess the degree of therapeutic change regarding psychological distress, psychosocial functioning and suicide risk. In addition, the application of CORE-SFB and VAS Pain in each session will allow to assess the trajectories of change experienced and the percentage of change session by session.

The proportion of patients who attain clinically significant improvement on the outcome measures (HDRS, HADS-D, HADS-A, FIQ and CORE-OM) will be calculated and compared between treatment conditions. Clinically significant change will be calculated considering the proposal of Jacobson and Truax. ${ }^{63}$ To test the differences in frequencies between the two intervention conditions, chi-square tests will be applied.

\section{Patient and Public Involvement}

The input received from patients participating in the pilot study $^{35}$ guided the design and the creation of the treatment protocols for the present study. In the current trial, the patients were not involved in the design of the study nor in the selection of the outcome measures.

\section{Discussion}

Depressive symptoms are present in a great proportion of patients with FM, these symptoms exacerbate its clinical and socio-economic consequences. Thus, depressive symptoms constitute one of the principal therapeutic targets for the treatment of patients with FM. Psychological treatments have demonstrated some efficacy in the treatment of depression in patients with FM. CBT, usually applied in group format, has accumulated enough evidence to be recommended as a treatment of choice in reviews and clinical guidelines; however, there is no evidence that this treatment acquires symptoms resolution. The limitations on reducing depressive symptoms in FM patients might be a product of the complexity of the syndrome and the resulting lack of treatment specificity. Then, an individual treatment that considers the needs and psychological profiles of each patient is needed. PCT might meet these requirements because it focuses on personal identity reconstruction and the meanings around significant others. One limitation of our study is that the generalization of the findings will be limited to women. Although FM is much more prevalent in women, the study will not provide any solid grounds to increase our knowledge about the treatment of this syndrome for male patients. Further studies are much needed in the future to address this issue.

\section{Conclusion}

The aim of this trial is to test the hypothesis that PCT might contribute to increase the efficacy in the reduction of depressive symptoms in patients with FM. We do not consider that cognitive conflicts are the cause or the principal factor of maintenance of depressive symptoms, but we believe that patients with dilemmas make depressive symptoms congruent with one part of their cognitive system (while it is undesirable for the other part). Whenever these dilemmas are detected, they might become the objective of the treatment. Cognitive conflicts are usually neglected by well-established treatments such as CBT.

By including in the research team members of different theoretical orientations (with extensive clinical experience in the treatment modalities being tested in the study), we aimed to minimize the potential allegiance bias described in the psychotherapy outcome research literature. If PCT reduces depressive symptoms, it might be considered as part of a multimodal approach, and recommended by the clinical guidelines. The efficacy of PCT as part of a multimodal treatment should be precisely gauged in future studies. 


\section{Abbreviations}

FM, Fibromyalgia; PCT, Personal Construct Therapy; CBT, Cognitive Behavioral Therapy; RGT, Repertory Grid Technique; HADS-D, Depression items of the Hospital Anxiety and Depression Scale; HADS-A, Anxiety items of the Hospital Anxiety and Depression Scale; HDRS, Hamilton Depression Rating Scale; FIQ, Fibromyalgia Impact Questionnaire; VAS Pain, Visual Analogue Scale for Pain; CORE-OM, Clinical Outcomes in Routine Evaluation Outcome Measure; CORE-SFB, Clinical Outcomes in Routine Evaluation - Short Form B; $t_{1}$, Time $1 ; t_{2}$, Time $2 ; t_{3}$, Time 3.

\section{Ethics and Dissemination}

This study has been approved by the Committee for Ethics in Research of the Universitat de Barcelona (IRB00003099) and by the ethical committees of the centers involved in the trial. The trial will be conducted in accordance with the Declaration of Helsinki.

The findings of the present trial will be presented in conferences and meetings of organizations interested in the treatment of FM, as well as in psychological interventions. All the results will be published in compliant peer-reviewed scientific journals and other information channels facilitating wider disseminations and general public knowledge.

Any deviation from the initial protocol registry will be recorded at ClinicalTrials.gov.

\section{Author Contributions}

All authors contributed to data analysis, drafting and revising the article, gave final approval of the version to be published, and agree to be accountable for all aspects of the work.

\section{Funding}

This research project is partially funded by the Ministry of Economy and Competitiveness (PSI2014-57957-R). Sponsor information: University of Barcelona, Gran Via de les Corts Catalanes, 58508007 Barcelona, Spain. The funding body and the sponsor do not have authority over the running of the trial and all decisions rest with the trial research team.

\section{Disclosure}

The authors report no conflicts of interest in this work.

\section{References}

1. Clauw DJ. Fibromyalgia. JAMA. 2014;311(15):1547. doi:10.1001/ jama.2014.3266
2. Queiroz LP. Worldwide epidemiology of fibromyalgia topical collection on fibromyalgia. Curr Pain Headache Rep. 2013;17:8. doi:10.1007/ s11916-013-0356-5

3. Branco JC, Bannwarth B, Failde I, et al. Prevalence of fibromyalgia: a survey in Five European Countries. Semin Arthritis Rheum. 2010;39(6):448-453. doi:10.1016/j.semarthrit.2008.12.003

4. Mas AJ, Carmona L, Valverde M, Ribas B, EPISER Study Group. Prevalence and impact of fibromyalgia on function and quality of life in individuals from the general population: results from a nationwide study in Spain. Clin Exp Rheumatol. 2008;26(4):519-526.

5. Gamero Ruiz F, Gabriel Sánchez R, Carbonell Abello J, Tornero Molina J, Sánchez-Magro I. El dolor en las consultas de Reumatología españolas: estudio epidemiológico EPIDOR [Pain in Spanish Rheumatology outpatient offices: EPIDOR epidemiological study]. Rev Clínica Española. 2005;205(4):157-163. doi:10.1157/13074161

6. Walker E, Keegan D, Gardner G, Sullivan M, Katon WJ, Bernstein D. Psychosocial factors in fibromyalgia compared with rheumatoid arthritis. Psychosom Med. 1997;59(6):565-571. doi:10.1097/00006842-199711000-00002

7. Arnold LM, Hudson J, Keck PE, Auchenbach M, Javaras K, Hess E, Comorbidity of fibromyalgia and psychiatric disorders. $J$ Clin Psychiatry. 2006;67(1):1219-1225. doi:10.4088/JCP.v67n0807

8. Veltri A, Scarpellini P, Piccinni A, et al. Review methodological approach to depressive symptoms in fibromyalgia patients. Exp Rheumatol. 2012;1(12):136-142.

9. Lange M, Petermann F. Einfluss von Depression auf das Fibromyalgiesyndrom. Der Schmerz. 2010;24(4):326-333. doi:10.1007/s00482-010-0937-8

10. Ozcetin A, Ataoglu S, Kocer E, et al. Effects of depression and anxiety on quality of life of patients with rheumatoid arthritis, knee osteoarthritis and fibromyalgia syndrome. West Indian Med J. 2007;56(2):122-129. doi:10.1590/S0043-31442007000200004

11. Finset A, Wigers SH, Gotestam KG. Depressed mood impedes pain treatment response in patients with fibromyalgia. $J$ Rheumatol. 2004;31(5):976-980.

12. Robinson R, Birnbaum H, Sisitsky T, Greenberg P, Wolfe F. Depression and fibromyalgia: treatment and cost when diagnosed separately or concurrently. Value Heal. 2003;6(3):357. doi:10.1016/ S1098-3015(10)64241-6

13. Alegre C, García J, Tomás M, et al. Documento de Consenso interdisciplinar para el tratamiento de la fi bromialgia [Intedisciplinary consensus document for the treatment of fibromyalgia]. Actas Españolas Psiquiatr. 2010;38(2):108-120.

14. Arnold LM, Clauw DJ, Jean Dunegan L, Turk DC. A framework for fibromyalgia management for primary care providers. Mayo Clin Proc. 2012;87(5):488-496. doi:10.1016/j.mayocp.2012.02.010

15. Goldenberg DL, Burckhardt C, Crofford L. Management of fibromyalgia syndrome. JAMA. 2014;292(19):2388-2395. doi:10.1001/ jama.292.19.2388

16. Glombiewski J, Sawyer AT, Gutermann J, Koenig K, Rief W, Hofmann SG. Psychological treatments for fibromyalgia: a meta-analysis. Pain. 2010;151(2):280-295. doi:10.1016/j. pain.2010.06.011

17. Bernardy K, Füber N, Köllner V, Häuser W. Efficacy of cognitive-behavioral therapies in fibromyalgia syndrome a systematic review and metaanalysis of randomized controlled trials. $J$ Rheumatol. 2010;37(10):1991-2005. doi:10.3899/jrheum.10 0104

18. Morley S, Williams A, Eccleston C. Examining the evidence about psychological treatments for chronic pain: time for a paradigm shift? Pain. 2013;154(10):1929-1931. doi:10.1016/j.pain.2013.05.049

19. Flor H, Turk DC. Chronic Pain: An Integrated Biobehavioural Approach. Seattle: IASP; 2011.

20. Vlaeyen JWS, Morley S, Cognitive-behavioral treatments for chronic pain: what works for whom? Clin J Pain. 2005;21(1):1-8. doi:10.1097/00002508-200501000-00001 
21. Van Houdenhove B, Luyten P. Customizing treatment of chronic fatigue syndrome and fibromyalgia: the role of perpetuating factors. Psychosomatics. 2008;49(6):470-477. doi:10.1176/appi.psy.49.6.470

22. Fitzcharles MA, Ste-marie PA, Goldenberg DL, et al. 2012 Canadian Guidelines for the Diagnosis and Management of Fibromyalgia Syndrome: Executive Summary. Pain Res Manag. 2013;18 (3):119-126. doi:10.1155/2013/918216.

23. Kelly GA. The Psychology of Personal Constructs. New York, NY: Norton; 1955.

24. Winter D, Viney L. Personal Construct Psychotherapy: Recent Advances in Theory, Practice and Research. London: Whurr/Wiley; 2005.

25. Feixas G, Villegas M. Constructivismo y Psicoterapia [Constructivism Ans Psychotherapy]. Bilbao: Desclée de Brouwer; 2000.

26. Metcalfe C, Winter D, Viney L. The effectiveness of personal construct psychotherapy in clinical practice: a systematic review and meta-analysis. Psychother Res. 2007;17(4):431-442. doi:10.1080/ 10503300600755115

27. Holland JM, Neimeyer RA, Currier JM, Berman JS. The efficacy of personal construct therapy: a comprehensive review. J Clin Psychol. 2007;63(1):93-107. doi:10.1002/jclp.20332

28. Feixas G, Cornejo JM. Manual de La Técnica de La Rejilla Mediante El Programa RECORD V.2.0 [Repertory Grid Technique Manual Using RECORD V.2.0]. Barcelona: Paidós; 1996.

29. Fransella F, Bell R, Bannister D. A Manual for Repertory and Technique. Chichester: John Wiley \& Sons, Ltd; 2004.

30. Feixas G, Erazo-Caicedo MI, Harter SL, Bach L. Construction of self and others in unipolar depressive disorders: a study using repertory grid technique. Cognit Ther Res. 2008;32(3):386-400. doi:10.1007/ s10608-007-9149-7

31. Compañ V, Feixas G, Varlotta-Domínguez N, et al. Cognitive factors in fibromyalgia: the role of self-concept and identity related conflicts. $J$ Constr Psychol. 2011;24(1):56-77. doi:10.1080/10720537.2 011.530492

32. Aguilera M, Paz C, Compañ V, Medina JC, Feixas G. Cognitive rigidity in patients with depression and fibromyalgia. Int $J$ Clin Heal Psychol. 2019;19(2):160-164. doi:10.1016/j.ijchp.2019.02.002

33. Montesano A, López-González MA, Saúl LÁ, Feixas G. A review of cognitive conflicts research: a meta-analytic study of prevalence and relation to symptoms. Neuropsychiatr Dis Treat. 2015;12:2997-3006. doi:10.2147/NDT.S91861

34. Feixas G, Montesano A, Compañ V, et al. Cognitive conflicts in major depression: between desired change and personal coherence. Br J Clin Psychol. 2014;53(1):369-385. doi:10.1111/bjc.12050

35. Aguilera M, Compañ V, Feixas G. Maltrato y reconstrucción de la identidad en la psicoterapia constructivista de la fibromialgia [Maltreatment and self-reconstruction in constructivist therapy for fibromyalgia]. Rev Argentina Clínica Psicológica. 2018;27:436443. doi:10.24205/03276716.2018.1056

36. Feixas G, Saúl LA. The multi-center dilemma project: an investigation on the role of cognitive conflicts in health. Span J Psychol. 2004;7(1):69-78. doi:10.1017/S1138741600004765

37. Feixas G, Compañ V. Dilemma-focused intervention for unipolar depression: a treatment manual. BMC Psychiatry. 2016;16(1):235. doi:10.1186/s12888-016-0947-x

38. Feixas G, Bados A, García-Grau E, et al. Efficacy of a dilemma-focused intervention for unipolar depression: study protocol for a multicenter randomized controlled trial. Trials. 2013;14(1):144. doi:10.1186/1745-6215-14-144

39. Feixas G, Bados A, García-Grau E, et al. A dilemma-focused intervention for depression: a multicenter, randomized controlled trial with a 3-month follow-up. Depress Anxiety. 2016;33(9):862-869. doi:10.1002/da.22510

40. Zigmond AS, Snaith RP. The hospital anxiety and depression scale. Acta Psychiatr Scand. 1983;67(6):361-370. doi:10.1111/j.16000447.1983.tb09716.x
41. Comeche MI, Martín A, Rodríguez M, Ortega J, Díaz M, Vallejo MÁ. Tratamiento Cognitivo-Conductual, Protocolizado y en Grupo, de la Fibromialgia [Group Cognitive-Behavioral Therapy protocol for fibromyalgia patients]. Clínica y Salud. 2010;21 (2):107-121. doi:10.5093/cl2010v21n2a1

42. Dimidjian S, Martell C, Addis M, Herman-Dunn R. Behavioral activation for depression. In: Clinical Handbook of Psychological Disorders. A Step-By-Step Treatment Manual. 4th ed. New York, NY: Guilford Press; 2008:328-364.

43. Beck J. Cognitive Behavior Therapy: Basics and Beyond. 2nd ed. New York, NY: Guilford; 2011.

44. Winter D, Procter H. Formulation in personal and relational construct psychology: seeing the world through clients' eyes. In: Johnston L, Dallos R, editors. Formulation in Psychology and Psychotherapy: Making Sense of People's Problems. Routledge; 2013:165-192.

45. Melzack R, Wall PD. Pain mechanisms: a new theory. Science. 1965;150(3699):971-979. doi:10.1126/science.150.3699.971

46. Duhl FJ, Kantor D, Duhl BS. Learning space, and action in family therapy: a primer of sculpture. Semin Psychiatry. 1973;5(2):167-183.

47. Feixas G, Saúl LA, Ávila-espada A. Viewing cognitive conflicts as dilemmas: implications for mental health. $J$ Constr Psychol. 2009;22 (2):141-169. doi:10.1080/10720530802675755

48. Terol M, López-Roig S, Rodríguez-Marín J. Propiedades psicométricas de la escala hospitalaria de ansiedad y depresión (HAD) en la población española [Psychometric properties of the hospital anxiety and depression scale (HAD) in the Spanish population]. Ansiedad y Estrés. 2007;13(2-3):163-176.

49. Vallejo M, Rivera J, Esteve-Vives J. Use of the Hospital Anxiety and Depression Scale (HADS) to evaluate anxiety and depression in fibromyalgia patients. Rev Psiquiatr y. 2012. 5(2):107-114.

50. Cabrera V, Martín-Aragón M, Del Terol MC, Núñez R, Pastor de Los MÁ. La Escala de Ansiedad y Depresión Hospitalaria (HAD) en fibromialgia: análisis de sensibilidad y especificidad [Hospital Anxiety and Depression Scale (HAD) in fibromyalgia: sensitivity and specificity analysis]. Ter Psicológica. 2015;33(3):181-193. doi:10.4067/S0718-48082015000300003

51. Nam S, Tin D, Bain L, Thorne JC, Ginsburg L. Clinical utility of the Hospital Anxiety and Depression Scale (HADS) for an outpatient fibromyalgia education program. Clin Rheumatol. 2014;33 (5):685-692. doi:10.1007/s10067-013-2377-1

52. Hamilton M. A rating scale for depression. J Neurol Neurosurg Psychiatry. 1960;23(1):56-62. doi:10.1136/jnnp.23.1.56

53. Ramos-Brieva J, Cordero A. Validación de la versión castellana de la escala de Hamilton para la depesión [Validation of the Spanish version of the Hamilton depression rating scale]. Actas LusoEspañolas Neurol psiquiátrica. 1986;14:324-334.

54. Burckhardt C, Clark S, Bennett R. The fibromyalgia impact questionnaire: development and validation. $J$ Rheumatol. 1991;18:728-733.

55. Esteve-Vives J, Rivera Redondo J, Isabel Salvat Salvat M, de Gracia Blanco M, de Miquel CA. Propuesta de una versión de consenso del Fibromyalgia Impact Questionnaire (FIQ) para la población española [Proposal for a consensus version of the Fibromyalgia Impact Questionnaire (FIQ) for the Spanish population]. Reumatol Clínica. 2007;3(1):21-24. doi:10.1016/S1699-258X(07)73594-5

56. Bennett R. The Fibromyalgia Impact Questionnaire (FIQ): a review of its development, current version, operating characteristics and uses. Clin Exp Rheumatol. 2005;23(Suppl. 39):S154-S162.

57. Huskisson EC. Measurement of pain. J Rheumatol. 1982;9 (5):768-769.

58. Hawker GA, Mian S, Kendzerska T, French M. Measures of adult pain: visual Analog Scale for Pain (VAS Pain), Numeric Rating Scale for Pain (NRS Pain), McGill Pain Questionnaire (MPQ), Short-Form McGill Pain Questionnaire (SF-MPQ), Chronic Pain Grade Scale (CPGS), Short Form-36 Bodily Pain Scale (SF). Arthritis Care Res (Hoboken). 2011;63(S11):S240-S252. doi:10.1002/acr.20543 
59. Evans C. Towards a standardised brief outcome measure: psychometric properties and utility of the CORE-OM. $\mathrm{Br} J$ Psychiatry. 2002;180(1):51-60. doi:10.1192/bjp.180.1.51

60. Trujillo A, Feixas G, Bados A, et al. Psychometric properties of the Spanish version of the clinical outcomes in routine evaluation - outcome measure. Neuropsychiatr Dis Treat. 2016;12:1457-1466. doi:10.2147/NDT.S103079

61. Feixas G, Cornejo JM GRIDCOR: correspondence analysis for grid data v.4.0 [Computer software and repertory grid manual]. 2002. www.terapiacognitiva.net/record.
62. Chan A-W, Tetzlaff JM, Altman DG, Dickersin K, Moher D. SPIRIT 2013: new guidance for content of clinical trial protocols. Lancet. 2013;381(9861):91-92. doi:10.1016/S0140-6736(12)62160-6

63. Jacobson N, Truax P. Clinical significance: a statistical approach to defining meaningful change in psychotherapy research. J Consult Clin Psychol. 1991;59(1):12-19. doi:10.1037/0022-006X.59.1.12

\section{Publish your work in this journal}

Neuropsychiatric Disease and Treatment is an international, peerreviewed journal of clinical therapeutics and pharmacology focusing on concise rapid reporting of clinical or pre-clinical studies on a range of neuropsychiatric and neurological disorders. This journal is indexed on PubMed Central, the 'PsycINFO' database and CAS, and is the official journal of The International Neuropsychiatric Association (INA). The manuscript management system is completely online and includes a very quick and fair peer-review system, which is all easy to use. Visit http://www.dovepress.com/testimonials.php to read real quotes from published authors. 